\title{
Effect of the Fiber Orientation Relatively to the Plasma Flow Direction in the Ablation Process of a Carbon-Phenolic Composite
}

\author{
Sônia Fonseca Costa e Silva ${ }^{1}$, Humberto Araújo Machado ${ }^{1,2}$, Edison Bittencourt ${ }^{3}$
}

\begin{abstract}
Composite materials are vastly employed in rocket nozzle extensions and as thermal protection shields in aerospace industry. In both cases, ablative and thermal properties play an important role for material choice and equipment sizing. These properties are strongly dependent on the way the pieces are manufactured. For the case of nozzle extensions, wrapping is the most common technique employed, which can be done by diverse ways. In this work, samples of the carbon reinforced carbon fiber (CRCF) were extracted from pre-forms obtained by biased and parallel tape wrapping processes with a resol-type phenolic resin used as polymer matrix, in order to study the influence of the fiber angle in the ablative behavior and thermal conductivity. Experimental results for both manufacturing techniques were obtained through ablation tests carried out in a plasma torch, were compared and had the different behaviors discussed. A computational simulation based in a two-front ablation model was also performed, in order to explain the influence of thermal conductivity on the different behaviors presented for biased fiber samples. Results show that the angle of the fibers relative to the heat flux direction is an important parameter to determine the ablative properties and the thermal performance of composite wrapped structures.
\end{abstract}

KEYWORDS: Ablation, Carbon-phenolic, Plasma torch, Computational simulation.

\section{INTRODUCTION}

The thermal resistance and ablation characteristics of composite materials are properties which play a very important role in the field of aerospace technology. When composite materials with excellent characteristics are exposed to extremely high temperatures, above $1,500^{\circ} \mathrm{C}$, not only their surface and structure but also their intrinsic features can be protected from the decomposition, which can occur at these temperatures. The ablation of a material depends on its intrinsic conditions and also on extrinsic conditions such as thermal, chemical and mechanical factors according to the variables of theoretical or practical environments (Schmidt, 1971). Ablation is an endothermic and erosive process which involves complex physical and chemical reactions.

There are several types of materials used in these ablative thermal protection systems, as each material presents certain advantages and disadvantages in terms of its properties (specific mass, mechanical strength, melting temperature etc.) and environmental conditions that they are subjected to (heat flow, temperature, mechanical stress etc.).

Carbon-based materials have been considered to be promising candidate for severe environments with super high temperature and high pressure for their high temperature capability (over $2,000^{\circ} \mathrm{C}$ ), high thermal shock resistance, good ablation resistance and low density (Kanevce et al., 1999).

Phenolic resin reinforced with carbon fabric has been a popular ablative material for rocket nozzles. Phenolic resin is the preferred matrix material due to its ability to efficiently

1. Instituto de Aeronáutica e Espaço - São José dos Campos/SP - Brazil 2.Universidade Estadual do Rio de Janeiro - Resende/RJ - Brazil 3.Universidade Estadual de Campinas - Campinas/SP - Brazil.

Author for correspondence: Sonia Fonseca Costa e Silva | Instituto de Aeronáutica e Espaço | Praça Marechal Eduardo Gomes, 50 - Vila das Acácias | CEP: $12.228-904$ São José dos Campos/SP - Brazil | Email: sonia_sfcs@hotmail.com

Received: 11/05/2014 | Accepted: 02/01/2015 
form char during endothermic pyrolysis (Tick et al., 1965). Carbon-fabric is preferred as reinforcement due to its ability to resist high-temperature erosive aerodynamic shear forces because of its high thermal stability, low thermal conductivity and higher inter-laminar shear strength (ILSS) in the composite form (Savage, 1993). However, there is always an urge to improve the ablation resistance of carbon-phenolic composites to further realize relatively thinner ablative composite structures to reduce the overall weight of aerospace systems.

Carbon fiber reinforced phenolic matrix composites (FRFC) have been used in the aerospace industry for the thermal-structural components. These materials can support aggressive thermal environments, such as in rocket nozzles throats, where a large amount of hot gases, due to the burning of propellant, pass through. These rocket nozzle extensions must submit, when in operation, low wear ablation on the inner walls. Furthermore, the wear should occur fairly homogeneous, i.e. distributed over the entire inner surface of the structure that has direct contact with the exhaust gases.

The carbon-phenolic ablative behavior should be influenced by the manufacturing process. The carbon-phenolic nozzle are commonly produced by a wrapping process, which consists in wrapping the carbon fiber tissue around a conic or bell shaped guide, in order to obtain the nozzle profile. The winding process with woven tape ("tape wrapping") basically consists on wrapping a tape over a steel mandrel. These strips are obtained by cutting fabric-based glass pre-impregnated with phenolic resin fiber, silica or carbon (Clements and Ward, 1966). The winding system is equipped with a compactor roller with pneumatic actuation, which compresses the "prepreg" tape as it is being deposited on the mandrel, as shown in Fig. 1.

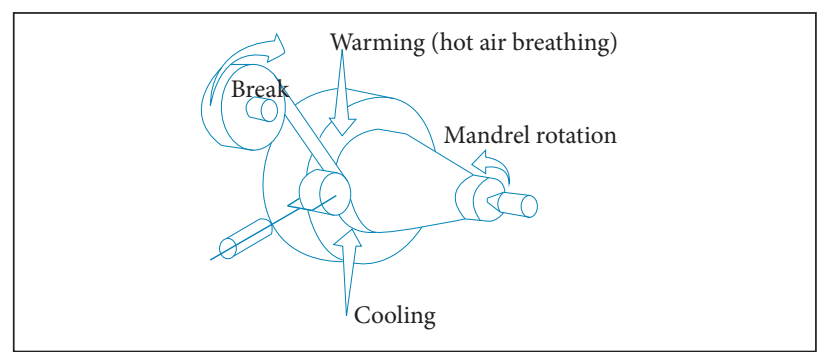

Figure 1. Scheme of a typical tape wrapping equipment.

The tape wrapping technique can be divided into:

- Parallel wrapping: consists on wrapping the tapes in the direction parallel to the main axis of the winding mandrel, as shown in Fig. 2. In this technique, the tapes used typically have fiber oriented towards $0^{\circ}$ and $90^{\circ}$ in a balanced manner, i.e. with an equal amount of filaments per unit length in the warp direction (the longitudinal direction of the fabric) and weft (transverse direction of the fabric), compared to the same principal directions (Clements and Ward, 1966). There is no pattern of weaving preferred for the tapes to be wounded in parallel. Figure 2 shows a plan view of the winding parallel deposition system showing the movement of the equipment's axes operating on a mandrel in the shape of a truncated cone.

- Biased wrapping: consists on wrapping the tapes in an angle (b) relative to the main axis of the winding mandrel direction (Clements and Ward, 1966). Compared to the parallel winding, this technique presents major practical difficulties. Most of the technological problems of this type of process arise from the fact that a single tape, at one point, has to go through different perimeters along the width, as evidenced in Fig. 3. For this to be possible in practice, the tapes to be used should allow a certain relative movement between the various fibers that constitute them. The fibers in this case should be directed to $\pm 45^{\circ}$ regarding the same principal directions and, as in the previous case, these fibers should be distributed

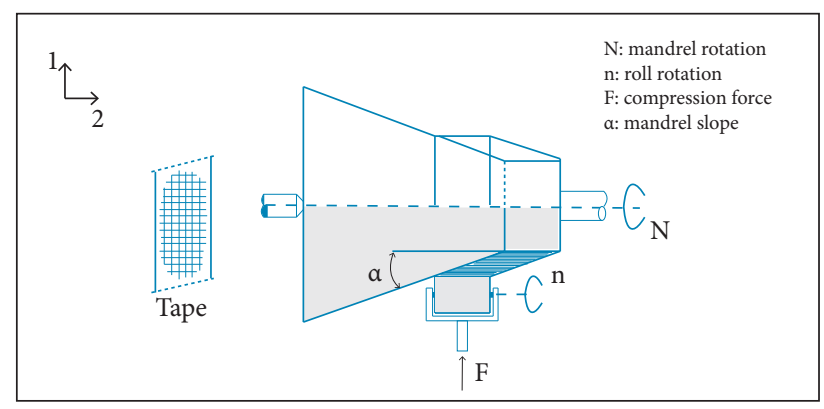

Figure 2. Parallel wrapping on conical mandrel.

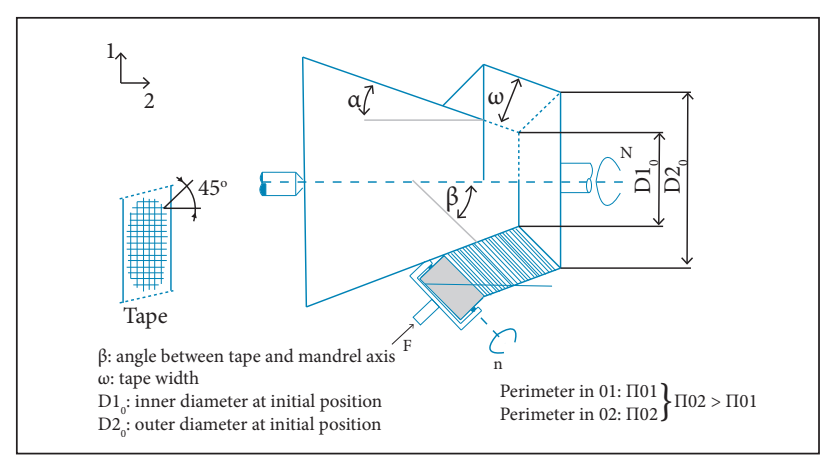

Figure 3. Biased wrapping on conical mandrel. 
in a balanced way in the weft (direction 2) and warp directions (direction 1).

For optimum levels of quality and performance of ablation be achieved, it is necessary that the polymerization of the composite be carried out under high pressure. For the polymerization of ablative high-performance composites, such as those proposed for the structure of the divergent nozzle and components, it is strongly recommended that more specialized equipment be used like hydroclaves, where the pressurizing medium is water in its liquid state (Clements and Ward, 1966).

In this paper, thermal degradation experiments were carried out using high flow of plasma jet (Fujimoto et al., 2003), in order to simulate the warmed gas flow through a rocket nozzle engine. The experimental results were obtained for three different angles of carbon fiber in test samples. A computational simulation is also performed, in order to compare the accuracy between the theoretical model for composite ablation and the empirical data.

\section{EXPERIMENTAL PROCEDURE MATERIALS}

The carbon fiber used in the present work is T22R-ECHO with thickness of $0.45 \mathrm{~mm}$, weave pattern $2 \times 2$ Twill, areal weight of $350 \pm 35 \mathrm{~g} / \mathrm{m}^{2}$ and density of $1.55 \mathrm{~g} / \mathrm{cm}^{3}$. A phenolic resin resol-type matrix with viscosity of $1,000 \mathrm{cPs}$ at $20^{\circ} \mathrm{C}$ has been used. The "prepreg" process was carried out in the Plastflow company facilities. After wetting, the fiber volume reached $55-60 \%$.

The inclined and parallel wrapping process was performed on a property equipment of the Instituto de Aeronáutica e Espaço (IAE) of the Departamento de Ciência e Tecnologia Aeroespacial (DCTA), The polymerization cycle was carried out in an hydroclave at high pressure, which gives the perfect polymerization of the phenolic resin used and an ablative structure with high compaction and low void content (porosity). The cycle used for polymerization (curing) was: $1 \mathrm{~h}$ at $100^{\circ} \mathrm{C}$ and $3 \mathrm{~h}$ at $165^{\circ} \mathrm{C}$ with a pressure of $7.0 \mathrm{MPa}$.

After the wrapping and curing process, the samples were extracted in different positions, according to the manufacturing process. Both the position of extraction and the wrapping process play important roles in the thermal properties and ablative behavior of the composite, since they directly affect the fiber orientation in the sample (Fig. 4), where, for parallel wrapping, $\alpha=22^{\circ}$ and, for biased wrapping, $\beta=20^{\circ}$.

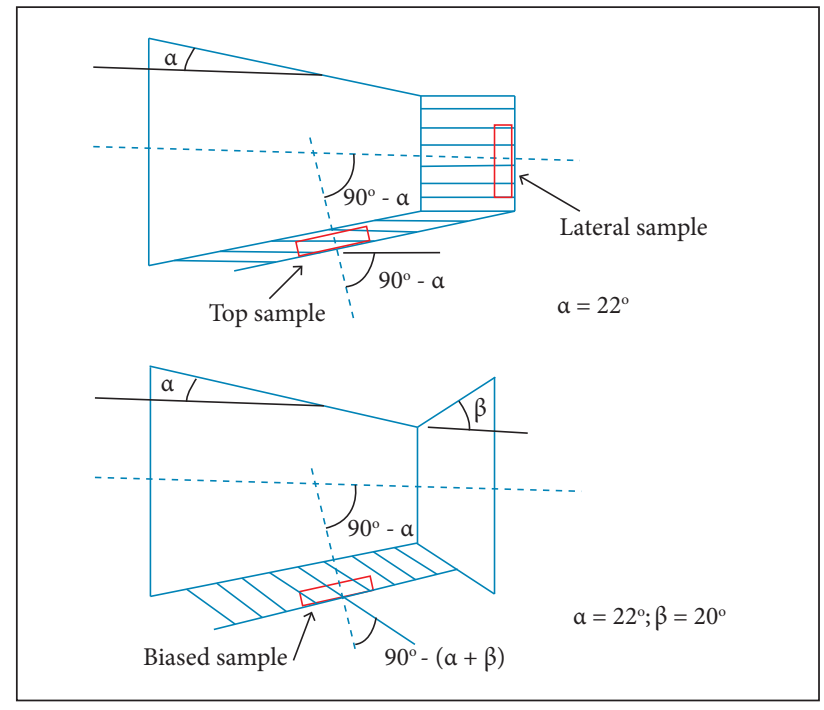

Figure 4. Positions for sample extraction.

\section{ABLATION TEST}

A plasma torch test was performed to investigate the ablation property of carbon-phenolic composites. The operation was carried out in atmospheric pressure. A direct current (DC) arc plasma system was designed for continuous working at a power up to $50 \mathrm{KW}$. The intensity of current was adjusted to $135 \mathrm{~A}$ with tension of $300 \mathrm{~V}$. So that, the maximum achievable power obtained, due the power supply, is about $30 \mathrm{KW}$, which gives a plasma enthalpy of about $5.5 \mathrm{MJ} / \mathrm{kg}$. The gas flow was maintained at $4.5 \times 10^{-3} \mathrm{~kg} / \mathrm{s}$. The specimen was placed vertically to the flame direction in air. Three fiber orientations were assessed in relation to exposure to plasma jet: parallel fibers to the plasma flow (parallel wrapping - lateral sample $-0^{\circ}$ ); fibers at an angle of $68^{\circ}$ in relation to the plasma flow (parallel wrapping - top sample); and fibers at an angle of $48^{\circ}$ in relation to the plasma flow (biased sample), as shown in Fig. 5.

The ablation test was carried out in a time from 30 to $120 \mathrm{~s}$ and the distance between the nozzle tip of the plasma gun and the front surface of the specimen varied from 4 to $10 \mathrm{~cm}$. The surface of the samples reached temperatures in the range from 1,200 to $1,900 \mathrm{~K}$ and it was measured by an optical pyrometer Mod. IR-AH 3SU Chino. Figure 6 shows the schematic illustration of the apparatus utilized for ablation test using an arc plasma torch.

The burn-through time was measured. The erosion rate was calculated by dividing the mass change before and after the test into a burn-through time for each specimen. One representative sample was used for each condition of testing. The specimens were cut in samples with diameter of $1.2 \mathrm{~cm}$. 


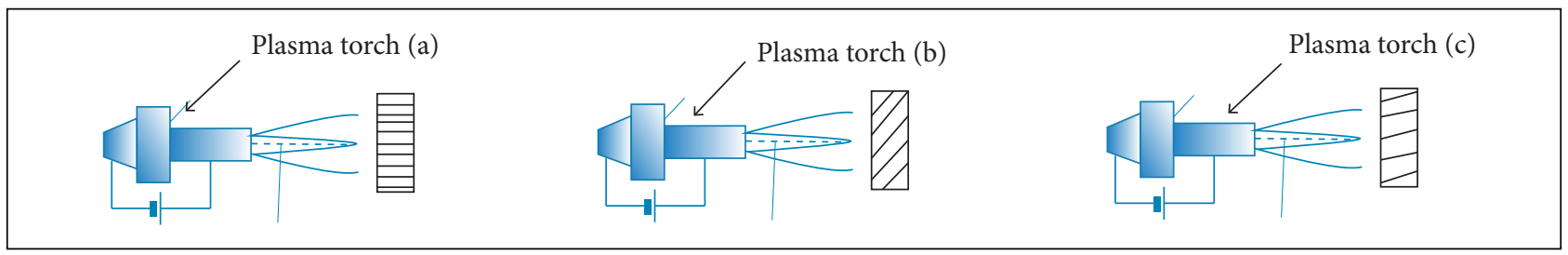

Figure 5. Orientation of the fibers in relation to the plasma flow: (a) parallel wrapping (lateral sample cut) - $0^{\circ}$, (b) parallel wrapping (top sample cut) - 68०, (c) biased wrapping (biased sample cut) - $48^{\circ}$.

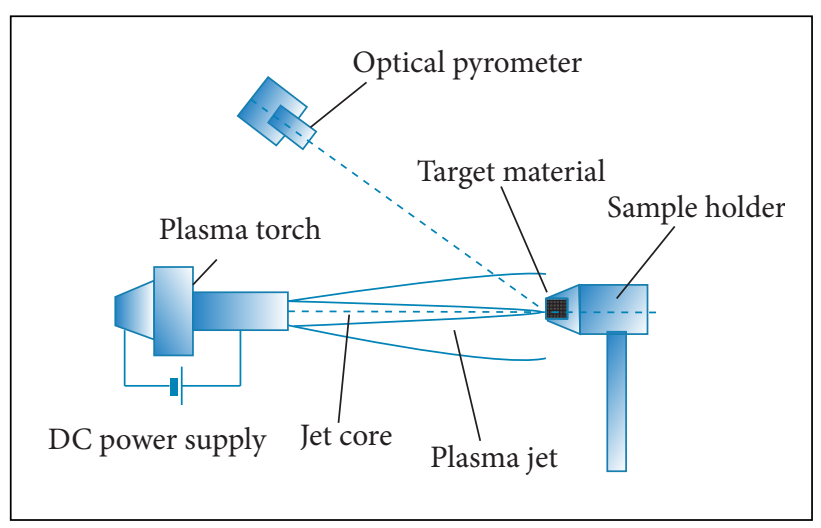

Figure 6. Scheme of an apparatus for ablation test using arc plasma torch.

\section{COMPUTATIONAL SIMULATION}

A computational code was employed to simulate a onedimensional ablation process, alike that conducted experimentally, where material samples were exposed to a plasma jet and had their temperature and heat flux measured. In this study, the target was not a perfect match between experimental and theoretical data but the qualitative behavior of the physical approach, in order to verify its coherence between the experimental and numerical results.

The ablation model proposed (Machado, 2014) for a composite material will consider the presence of two layers: the virgin material and the char layer that appears after the pyrolysis front. Two moving fronts will be accounted: the pyrolysis and the ablation front of the char layer. Both will appear at constant and specific temperatures. The hypotheses considered to build the mathematical model are:

- Solid materials are isotropic with constant properties.

- The pyrolysis front is considered to have zero thickness.

- Char layer recession is supposed to occur due oxidation or sublimation at constant temperature.

- Melting layer is absent.

- The resulting gases fully react and perfectly mix with the external air layer, without influence over its properties.
- $\quad$ Air is considered a perfect gas.

- Radiation is absorbed or emitted by the external surface, but is not transmitted.

The moving boundary problem was solved by the interface tracking method, introduced by Unverdi and Tryggvason (1992) and employed by Juric (1996) in the solution of phase-change problems. In this method, a fixed uniform Eulerian grid is generated, where the conservation laws are applied over the complete domain. The interface acts as a Lagrangian referential, where a moving grid is applied. The instantaneous placement of the interface occurs through the constant remeshing of the moving grid, and each region of the domain is characterized by the indicator function, which identifies the properties of the wall and the air around it. The method can be extended for various interfaces, through the use of one particular indicator function for every phase within the domain of simulation (Machado, 2013). This method allows the representation of any geometry and also the characterization of every layer separately. It is accomplished without a high increase in the computational cost and does not need any pre-processing (construction of unstructured grid or coordinate transformation). In this work, this method is employed to estimate the ablative performance of the carbon-phenolic composite, considering a two-dimensional approach in both the heat conduction and the moving boundary problem. Both the set of equations and the numerical solution are described in detail in the work of Machado (2014).

\section{RESULTS}

The specimens submitted to ablation test were extracted to pre-form obtained by biased and parallel wrapping using a hole saw to remove small cylinders with a diameter of $12.5 \mathrm{~mm}$. After cutting, each sample was identified and weighed on a precision balance $( \pm 0.0001 \mathrm{~g})$. A reweighing of the specimens was also taken after exposure to heat flow, to determine the rate of mass removed per unit of area and time of exposure to the plasma jet $\left(\mathrm{kg} / \mathrm{m}^{2} \mathrm{~s}\right)$ and to estimate the experimental 
mass loss rate. According to the results, the mass loss of the samples increases with time of exposure to the plasma jet in both manufacturing processes, biased and parallel wrapping (side section and top cut), as shown in Figs. 7, 8 and 9. The samples obtained by parallel wrapping with the side cut exposure had higher removing mass in relation to exposed samples cut from

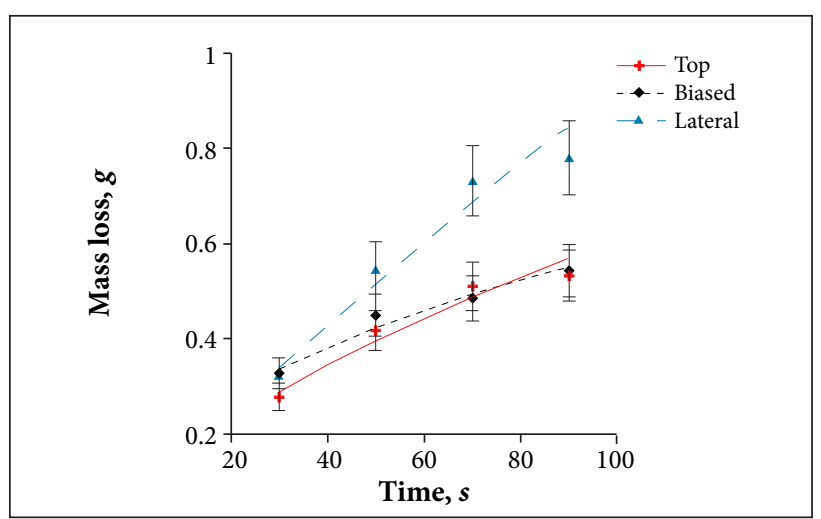

Figure 7. Variation of mass loss over time for a heat flux of $0.626 \mathrm{MW} / \mathrm{m}^{2}$.

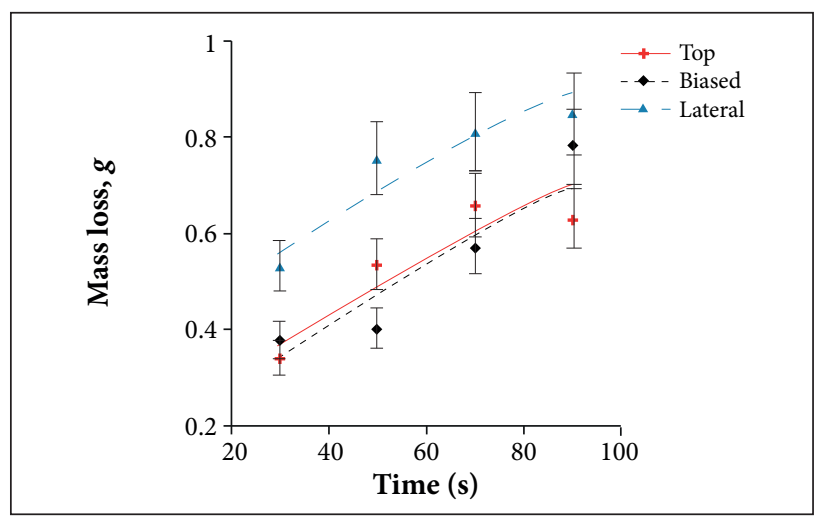

Figure 8. Variation of mass loss over time for a heat flux of $0.903 \mathrm{MW} / \mathrm{m}^{2}$.

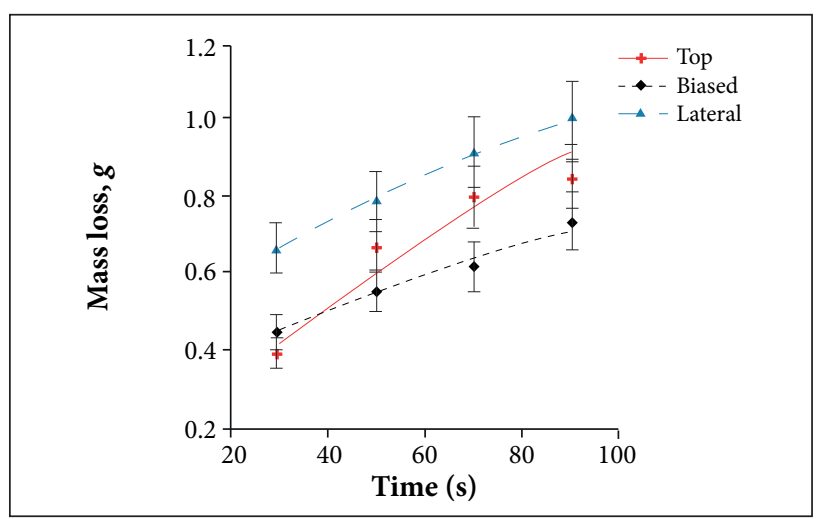

Figure 9. Variation of mass loss over time for a heat flux of $1.376 \mathrm{MW} / \mathrm{m}^{2}$. the top and samples obtained by biased wrapping, due to the orientation of the fibers.

If the fibers are oriented parallel to the plasma flow, high mechanical strength enhancement of the orientation plane is produced and a minimum internal heat conduction is also achieved because heat transfer occurs almost completely through the resin and, with strict packaging of the fibers, it becomes more difficult. The resulting effect is a high non-uniform ablation and the tendency to mechanical erosion is increased by gases formed in the substrate material, which are trapped by closely packed fibers and yield pressure rising, resulting in a crack in the material. We observed that the mass loss is lower in the samples with a certain angle relative to the plasma flow (Figs. 10 and 11).

Fibers oriented perpendicular to the direction of flow offer greater resistance to shear flow and minimize the strengthening mechanical surface erosion, reducing ablation speed and maximizing the amount of heat ablation compared to fibers oriented in other directions, because of the greater length of

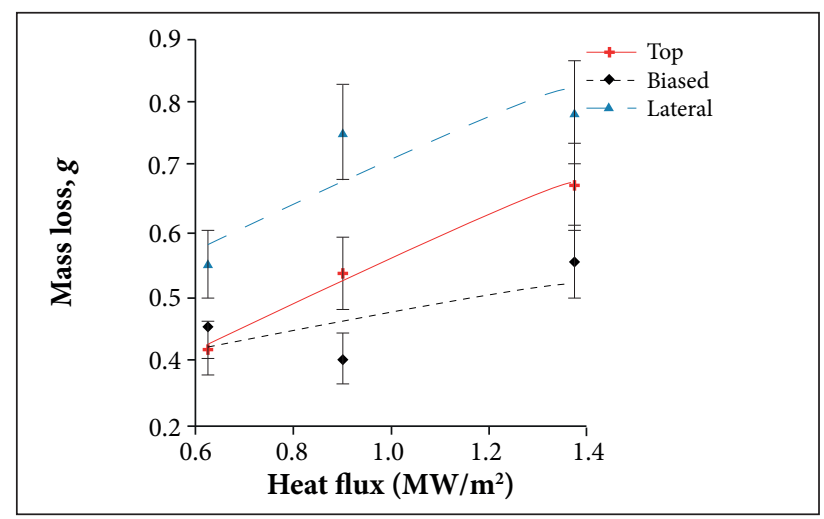

Figure 10. Results of the variation of total mass flow in the heat exposure time of $50 \mathrm{~s}$.

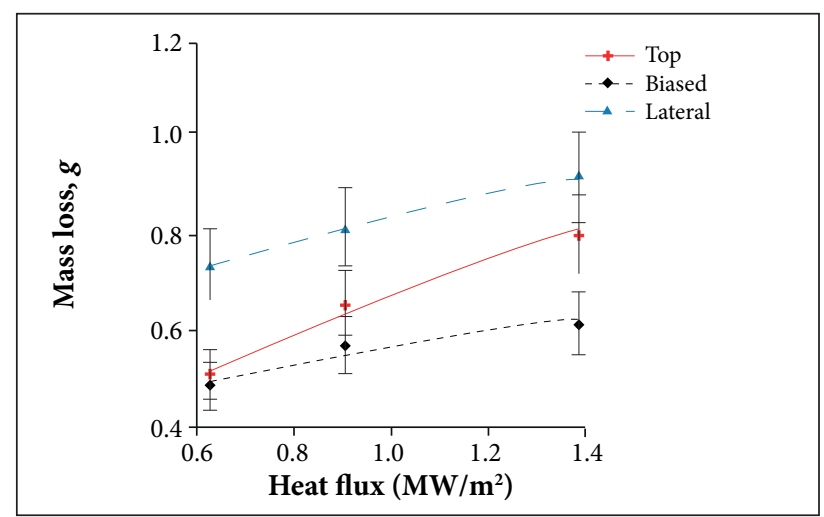

Figure 11. Results of the variation of total mass flow in the heat exposure time of $70 \mathrm{~s}$. 
continuous fiber supported by the phenolic resin, since it is not easily removed by the high-velocity gas flow, causing the material to be capable of presenting greater resistance to mechanical erosion. This erosion resistance regularly decreases as the fibers approach the parallel orientation. There are also basic limitations for this type of orientation. The mechanical properties are relatively low at the plane perpendicular to the fibers; this leads to occasional material removal and failure by stress during ablation. With the fibers in this orientation, the heat transfer rate of the substrate material can be appreciably higher because they provide a direct drive line to the inside, increasing the thickness of the material required to produce the desired insulation.

The mass loss rate was the parameter chosen in order to compare the experimental data and numerical results. The rate was obtained dividing the mass loss by the time of exposure. The same procedure was employed to obtain the mean mass loss rate in the simulations. However, the calculation of the mass loss rate is done instantaneously through the integration at each time step, while the experimental method shows the final result after the selected period.

The properties used in the simulation are presented in Table 1 (Machado, 2013). Although some of the thermophysical properties of the ablative materials were measured during the experiments, most of them had to be extracted from diverse literature

Table 1. Properties of the carbon-phenolic resin.

\begin{tabular}{|c|c|c|}
\hline Property & Virgin & Char \\
\hline $\mathrm{k}\left(\mathrm{W} / \mathrm{m}^{\circ} \mathrm{C}\right)$ & $0.8-11^{1}$ & $1.73^{2}$ \\
\hline $\mathrm{Cp}\left(\mathrm{J} / \mathrm{kg}^{\circ} \mathrm{C}\right)$ & $1,300^{1}$ & $1,800^{3}$ \\
\hline$\rho\left(\mathrm{kg} / \mathrm{m}^{3}\right)$ & $1,700^{1}$ & $1,184^{3}$ \\
\hline e & $0.92^{4}$ & $0.7^{3}$ \\
\hline Heat of pyrolysis $(\mathrm{MJ} / \mathrm{kg})$ & $2.33^{5}$ & \\
\hline Pyrolysis temperature $\left({ }^{\circ} \mathrm{C}\right)$ & $350^{6}$ & \\
\hline Heat of melting/sublimation (MJ/kg) & & $10.5^{3}$ \\
\hline Melting temperature $\left({ }^{\circ} \mathrm{C}\right)$ & & $3,700^{3}$ \\
\hline
\end{tabular}

Table 2. Surface temperature after 90 s warming. sources. The values for thermal conductivity depend on the fiber orientation. The higher value $\left(11 \mathrm{~W} / \mathrm{m}^{2 \circ} \mathrm{C}\right)$ is considered when the fiber is normal to the surface were the heat flux is imposed (parallel to the heat flux direction) and the lower value $\left(0.8 \mathrm{~W} / \mathrm{m}^{2 \circ} \mathrm{C}\right)$ is considered when the fibers lie parallel to the surface and normal to the heat flux direction. Both cases were considered for the numerical simulation.

The one-dimensional heat conduction problem was solved considering prescribed heat flux in the warmed face and adiabatic surface in the opposite side, as boundary conditions. The initial temperature was $27^{\circ} \mathrm{C}(300 \mathrm{~K})$. The same heat fluxes measured in the samples - $0.464 \mathrm{MW} / \mathrm{m}^{2}, 0.626 \mathrm{MW} / \mathrm{m}^{2}$, $0.903 \mathrm{MW} / \mathrm{m}^{2}, 1.376 \mathrm{MW} / \mathrm{m}^{2}$ and $1.725 \mathrm{MW} / \mathrm{m}^{2}$ - were imposed to the warmed face.

Table 2 shows the temperature of sample's surface in the side were the plasma jet was impinged (for the experiment) and the heat flux was imposed (simulation). Deviation of the numerical results relative to the average experimental data rises from -7.82 to $18.7 \%$. Even considering the uncertainty of the experimental results, the simple approach used in the mathematical model (that neglects the influence of various physical processes) and the values of the properties used in the calculation (which were extracted from diverse sources and consequently are subjected to different conditions of evaluation) are considered to present physical coherence and both curves, numerical and experimental, have a similar behavior, as shown in Fig. 12.

Figures 13, 14 and 15 show the comparison among the experimental results of all samples for each value of thermal conductivity used in the computational simulation and the mass loss rate with the time. The experimental results present the points resulting of measurements and the respective fitting curves, according to the slope of the fiber. In all cases, the mass loss rate seems to be underestimated by numerical simulation. The numerical results show little influence of thermal conductivity variation. The results for both values used $\left(0.8\right.$ to $\left.11 \mathrm{~W} / \mathrm{m}^{2 \circ} \mathrm{C}\right)$ are relatively close. In Fig. 13, the sample curves obtained by

\begin{tabular}{|c|c|c|c|c|c|}
\hline \multirow[t]{2}{*}{ Case } & \multicolumn{5}{|c|}{ Heat flux $\left(\mathrm{MW} / \mathrm{m}^{2 \circ} \mathrm{C}\right)$} \\
\hline & 0.464 & 0.626 & 0.903 & 1.376 & 1.725 \\
\hline Simulation $-\mathrm{k}=0.8 \mathrm{~W} / \mathrm{m}^{2} \mathrm{~K}$ & 1,113 & 1,342 & 1,624 & 1,942 & 2,097 \\
\hline Simulation $-\mathrm{k}=11 \mathrm{~W} / \mathrm{m}^{2} \mathrm{~K}$ & 1,070 & 1,321 & 1,620 & 1,944 & 2,103 \\
\hline Simulation - average & 1,091 & $1,331.5$ & 1,622 & 1,943 & 2,100 \\
\hline Parallel wrapping - top sample & 1,200 & 1,190 & 1,450 & 1,640 & 1,745 \\
\hline Parallel wrapping - lateral sample & 1,167 & 1,275 & 1,375 & 1,667 & 1,792 \\
\hline Parallel wrapping - average & $1,183.5$ & $1,232.5$ & $1,412.5$ & $1,653.5$ & $1,768.5$ \\
\hline$\%$ deviation (relative to experiment) & -7.82 & 8.03 & 14.8 & 17.5 & 18.7 \\
\hline
\end{tabular}


parallel wrapping (lateral and top sample) and biased wrapping produce the same slope compared with the numerical simulation, without crossing at any point. The crossing between the curves appears in all cases for numerical simulation, but only in the two last cases for the experimental results (Figs. 14 and 15). Although the first case does not present such crossing, these curves feature similar behavior of their numerical counterparts. In all cases, the lateral sample curves present a level of mass loss rate above the two other samples. It indicates that the influence of the fiber orientation and the thermal conductivity variation affects the ablation process.

When the fibers are disposed normally to the heat flux, conduction occurs mainly through the resin, and the result is a high and non-uniform ablation as described for each value of heat flux observed for the samples obtained by biased and top cut parallel wrapping. The samples where fibers are perpendicular or biased to the direction of gas flow provide greater resistance to cutting gas effort and minimize the mechanical erosion of the surface, reducing the ablation rate, as shown in Figs. 13,

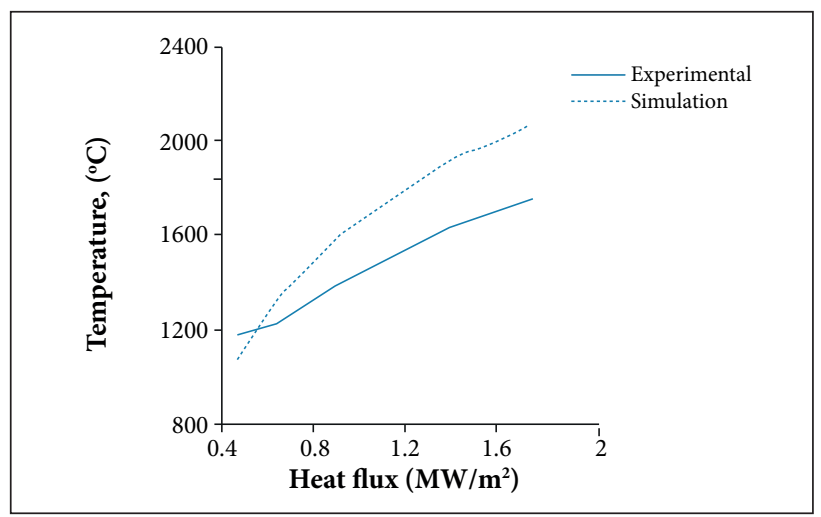

Figure 12. Temperature at $\mathrm{t}=90 \mathrm{~s}$ in the sample's warmed surface, according to heat flux.

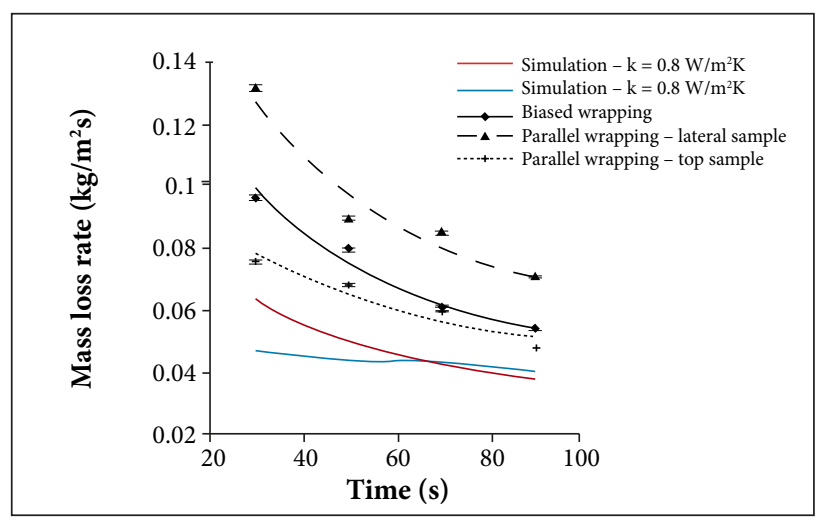

Figure 13. Mass loss rate with time: simulation and experimental results for $\mathrm{q}=0.626 \mathrm{MW} / \mathrm{m}^{2}$.
14 and 15 , which is confirmed by the proximity of both curves in all results.

The discrepancy between the experimental and numerical temperatures and mass loss rates can be the result of other processes. The catalycity (Carleton and Marinelli, 1992; Kovalev and Kolesnikov, 2005; Barbato et al., 2000) effect of the surface over the recombination of species produced by decomposition due to high temperature can increase the heat flux. Since this is an exothermic reaction, such increasing may have resulted in higher heating rates and mass loss rates, which is not considered in the numerical simulation.

Resin pyrolysis yields gaseous products that percolate toward the heated surface and are injected into the boundary layer which changes its properties, usually resulting in a reduction in convective heating. In addition, chemical reactions between the surface material of the boundary layer and pyrolysis-resulting species can result in consumption of the leading surface material and surface recession. These reactions can be endothermic (vaporization, sublimation) or

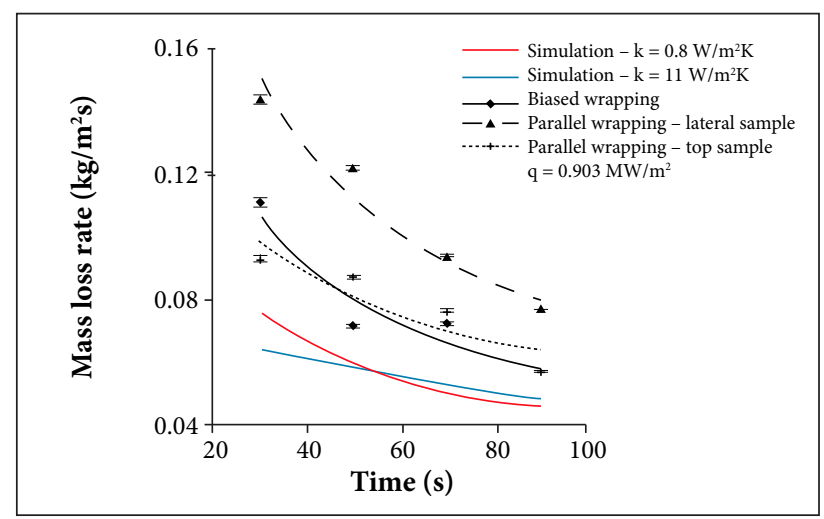

Figure 14. Mass loss rate with time: simulation and experimental results for $\mathrm{q}=0.903 \mathrm{MW} / \mathrm{m}^{2}$.

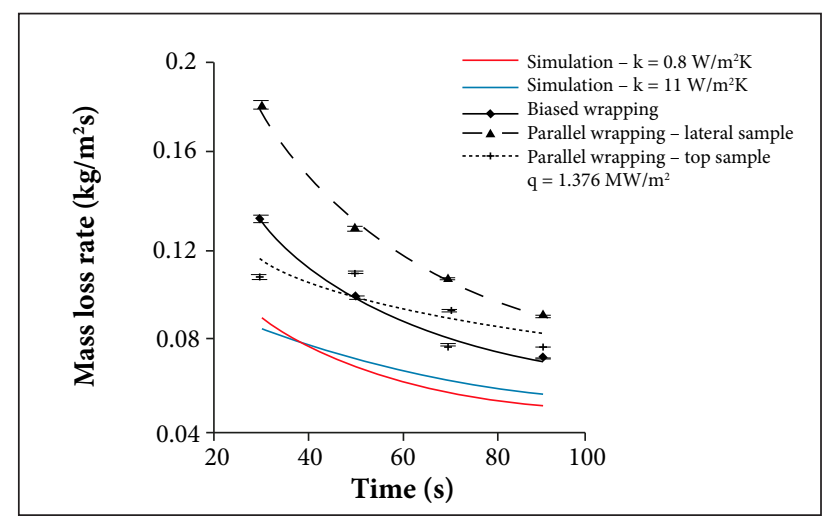

Figure 15. Mass loss rate with time: simulation and experimental results for $\mathrm{q}=1.376 \mathrm{MW} / \mathrm{m}^{2}$. 
exothermic (oxidation) and will have an important impact on energy to the surface.

These effects may result in increasing mass loss rate and decreasing temperature, when compared with the mathematical model. Such effects are implicit in the physical parameters that were measured under specific conditions, which are not exactly applicable to the actual physical phenomenon and occur at high temperatures. It is expected that these effects occur during the tests in plasma torch because the temperatures in samples' surface can reach values between $3,600^{\circ} \mathrm{C}-5,000^{\circ} \mathrm{C}$.

The intersection between the curves for the mass loss rate with time among the biased and top parallel wrapping processes appear to be mainly due to the thermal conductivity variation with the angle of inclination of the fibers, since the two curves are far from the lateral parallel wrapping curve in which the fibers are parallel to the heat flow; consequently, ablation processes involved are different from those in which the fibers are inclined in relation to the flow. When the experimental results are compared with the numerical simulation for the two extreme values of thermal conductivity $\left(0.8 \mathrm{~W} / \mathrm{m}^{2 \circ} \mathrm{C}\right.$ when the fiber is perpendicular to the flow and $11 \mathrm{~W} / \mathrm{m}^{2 \circ} \mathrm{C}$ when the fiber is parallel), results present the same behavior. It is noted that, as the heat flux increases, the crossover point tends to the left, in the direction of convergence of the curves on the initial times. A possible explanation for this behavior, based on numerical results, is related to the difference in conductivity between the carbonized layer ("char") and the virgin material. The "char" works as an insulator, with a conductivity slightly above the lowest value considered for the virgin material $\left(1.73 \mathrm{~W} / \mathrm{m}^{2 \circ} \mathrm{C}\right)$ and reaches higher temperatures than this, because it is directly exposed to the plasma jet and its maximum surface temperature (melting point) can reach $3,700^{\circ} \mathrm{C}$.

Figure 16 allows understanding the process. The first column shows the position of the pyrolysis front, which separates the virgin material and the char layer. In the case of lower conductivity, the heat flux incident on the surface takes longer to spread into the material and the pyrolysis temperature is reached earlier on a smaller thickness, starting the formation of char layer. In the case of higher conductivity, the heat spreads faster, yielding a lower temperature gradient, which delays the surface to achieve pyrolysis temperature. In both cases, when the pyrolysis temperature is reached, the velocity of recession of the pyrolysis front shoots to a peak and then reduces gradually, as seen in the right column of

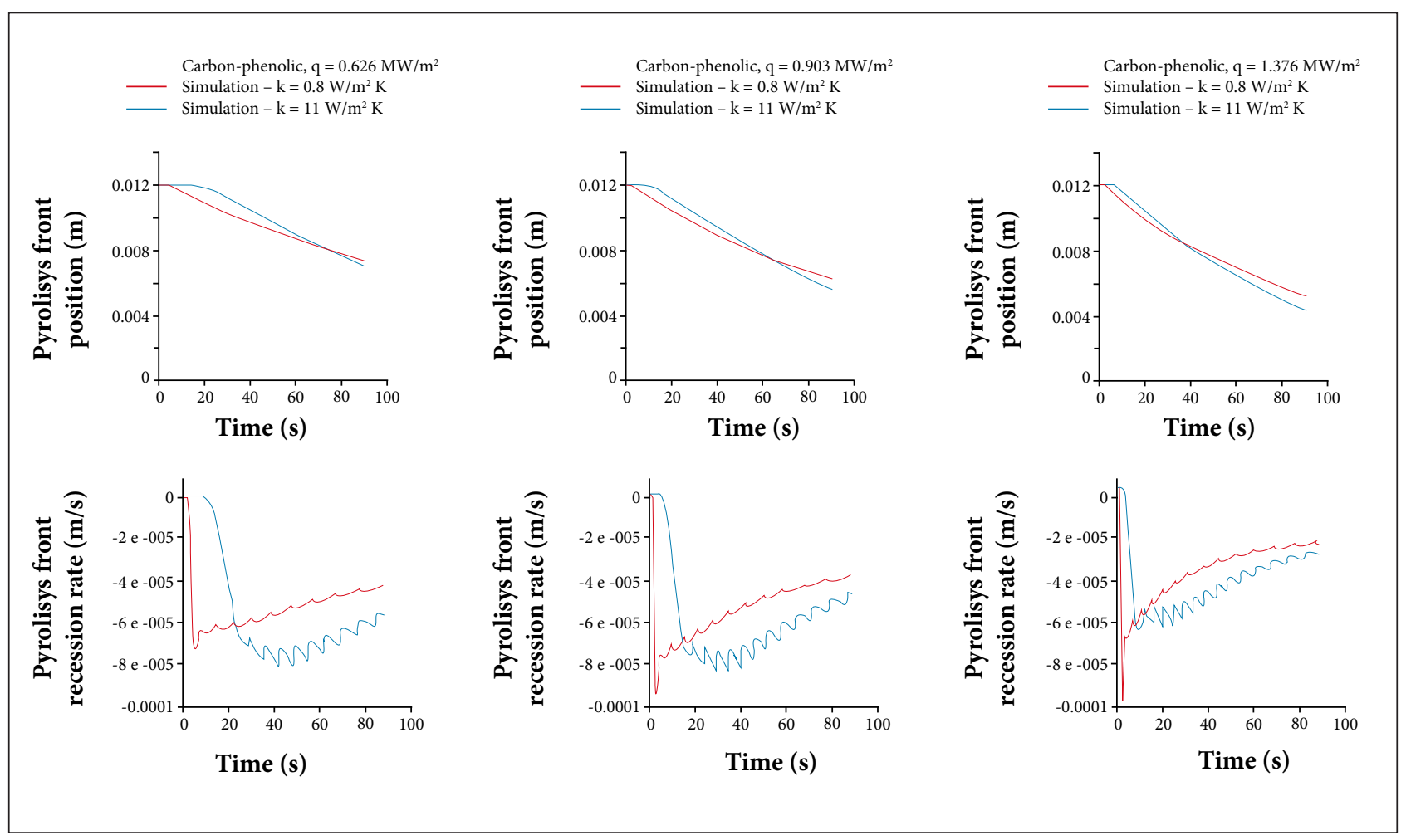

Figure 16. Effects of thermal conductivity difference between carbonized layer ("char") and the virgin material over the pyrolysis dynamics. 


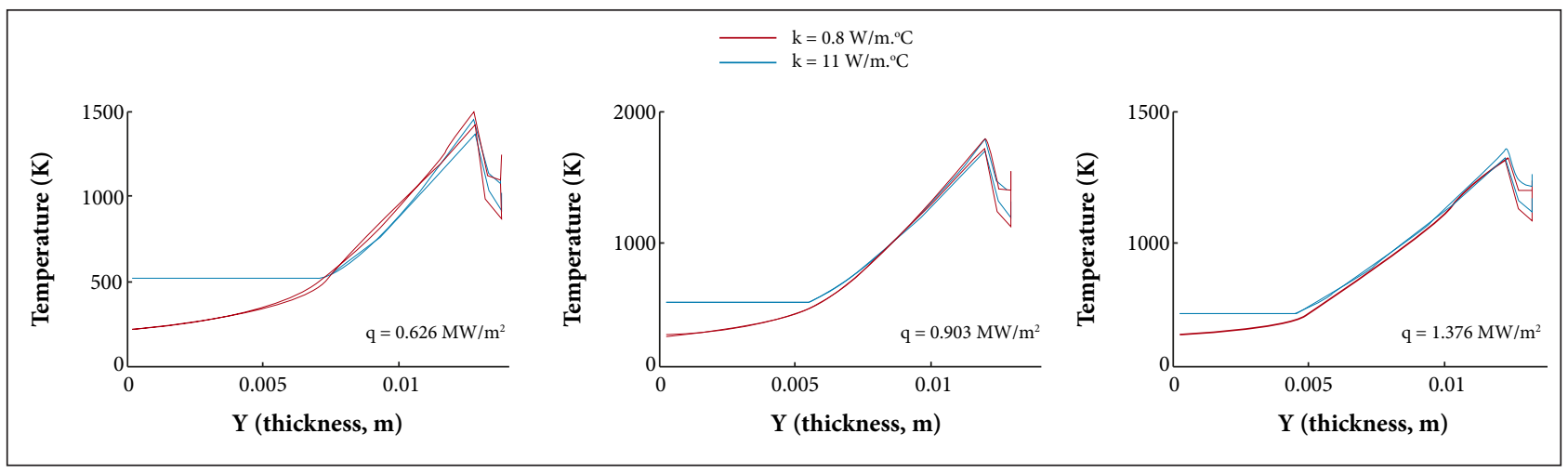

Figure 17. Temperature profile in the char and virgin layers of "char" after 90 s of jet exposure.

Fig. 16, with the resulting char layer working as a thermal insulator. In every case (high and low conductivity), the speed of pyrolysis fronts converge with the time and the difference between them reduces as heat flux increases.

Figure 17 shows results for temperature distribution after a period of $90 \mathrm{~s}$. In the case of lower thermal conductivity, the highest temperature gradients are observed in the virgin material layer, which results in lower temperatures at the opposite surface to the heat flux. In the case of higher thermal conductivity, there is almost no temperature gradient in the virgin material layer, resulting in a higher temperature on the opposite side to the heat flux. This fact is relevant for a thermal protection system, since one of the objectives of the ablative heat shield is to ensure that temperatures remain below the operating limits. In both cases, the temperature profile of the char layer is quite similar.

\section{CONCLUSION}

In this paper, the effect of the fiber orientation in a carbonphenolic ablator was studied. Experimental results were obtained from a test with a plasma jet emulating the gas flow within a rocket nozzle engine, using samples made by two different wrapping procedures and extracted from two different ways. The experimental data were compared with numerical results of computational simulation, obtained for the maximum and minimum values of thermal conductivity for the composite, according to the fiber orientation (perpendicular or parallel to the heat flux).

Comparison of surface temperature at $90 \mathrm{~s}$ of warming showed relatively good agreement between experiment and computational simulation, in spite of all uncertainties involved in both methods. The numerical results showed little effect of thermal conductivity over the mass loss rate. However, the experimental results showed a considerable difference between the top sample (fibers normal to heat flux) and the lateral and biased sample (fibers inclined to heat flux direction), which indicates that the fiber slope relative to the heat flux affects other physical processes involved in ablation, like pyrolysis gas releasing and surface catalycity effect.

Although the difference between numerical and experimental results and between the top samples and the lateral and biased samples, the order of magnitude and the curve behavior were considered similar, which attested the physical coherence of all results.

\section{REFERENCES}

Barbato, M., Reggiani, S., Bruno, C. and Muylaert, J., 2000, "Model for Heterogeneous Catalysis on Metal Surfaces with Applications to Hypersonic Flows", Journal of Thermophysics and Heat Transfer, Vol. 14, No. 3, pp. 412-420. doi: 10.2514/2.6539

Barros, E.A., 2008, "Thermal Plasma for Ablation in Materials Used in Heat Shield of Space Systems" (in Portuguese), Master's Dissertation, Instituto Tecnológico de Aeronáutica, São José dos Campos, Brazil.
Carleton, K.L. and Marinelli, W.J., 1992, "Spacecraft Thermal Energy Accommodation from Atomic Recombination", Journal of Thermophysics and Heat Transfer, Vol. 6, No. 4, pp. 650-655. doi: 10.2514/3.11547

Clements, H.R. and Ward, G.T., 1966, "Fabrication of Ablative Liners for Large Solid Booster Nozzles", Journal of Spacecraft and Rockets, Vol. 3, No. 4, p. 483-488. doi: 10.2514/3.28480 
Fujimoto, K., Shioya, T. and Satoh, K., 2003, "Degradation of CarbonBased Materials due to Impact of High-Energy Atomic Oxygen", International Journal of Impact Engineering, Vol. 28, No. 1, pp. 1-11. doi: 10.1016/S0734-743X[02)00057-X

Juric, D., 1996, "Computations of Phase Change", Ph.D. Thesis, University of Michigan, Ann Arbor, USA.

Kanevce, L.P., Kanevce, G.H. and Angelevski, Z.Z., 1999, "Comparison of Two Kinds of Experiments for Estimation of Thermal Properties of Ablative Composite", Proceedings of the $3^{\circ}$ International Conference on Inverse Problems in Engineering, Port Ludlow, USA

Khlybov, V.I. and Makhankov, S.A., 2008, "Experimental Studies to Determine Ablation Parameters and Emissivity of Thermal-Protective Materials", Technical Report, OAO-Makeyev GRT (Restricted).

Kovalev, V.L. and Kolesnikov, A.F., 2005, "Experimental and Theoretical Simulation of Heterogeneous Catalysis in Aerothermochemistry (a Review)", Fluid Dynamics, Vol. 40, No. 5, pp. 669-693. doi: 10.1007/s10697-005-0106-4

Machado, H.A., 2013, "Simulation of Ablation in a Sounding Rocket Thermal Protection System Via an Interface Tracking Method with Two Moving Fronts", Journal of Aerospace Technology and Management, Vol. 5, No. 4, pp. 409-420. doi: 10.5028/jatm.v5i4.262

Machado, H.A., 2014, "Simulation of Two Moving Fronts Ablation in a Composite Thermal Protection System Via an Interface Tracking
Method", Proceedings of the $11^{\circ}$ IAAA/ASME Joint Thermophysics and Heat Transfer Conference, Atlanta, USA.

Russel, G.W., 1996, "Evaluation of Thermal of an Epoxy Composite Using a Complex Modeling Technique", Proceedings of the AIAA, ASME and ASEE, $32^{\circ}$ Joint Propulsion Conference and Exhibit, Lake Buena Vista, USA.

Savage, G., 1993, "Carbon-Carbon Composites", Chapman \& Hall, London, 389p.

Savvatimskii, A.V., 2003, "Melting Point of Graphite and Liquid Carbon", Physics-Uspekhi, Vol. 46, No. 12, pp. 1295-1303. doi: 10.1070/PU2OO3v046n12ABEH001699

Schmidt, D.L., 1971, "Ablative Polymers in Aeroespace Technology", In: D'Alelio, G.F. and Parker, J.A. [Eds.), "Ablative Plastics", Marcel Dekker, New York, USA, pp. 1-2.

Sutton, K., 1970, "An Experimental Study of a Carbon-Phenolic Ablation Material”, NASA Technical Note D-5930.

Tick, S.J., Huson, G.R. and Griese, R., 1965, "Design of Ablative Thrust Chambers and Their Materials", Journal of Spacecraft and Rockets, Vol. 2, No. 3, pp. 325-331. doi: 10.2514/3.28179

Unverdi, S.O. and Tryggvason, G., 1992, "A Front-Tracking Method for Viscous, Incompressible, Multi-fluid Flows", Journal of Computational Physics, Vol. 100, No. 1, pp. 25-37. doi: 10.1016/00219991(92)90307-K 